\title{
Small Animal PET as Non-Invasive Tool for Preclinical Imaging
}

\author{
M. Oteo* AND M.A. MORCILlO \\ Biomedical Applications and Pharmacokinetics Unit, CIEMAT, Avda. Complutense 40, 28040 Madrid Spain
}

\begin{abstract}
Increasingly, in vivo imaging of small laboratory animals has become critical for preclinical biomedical research allowing the non-invasive assessment of biological and biochemical processes in living subjects. Many different positron labeled compounds have been synthesized as tracer that target a range of specific markers or pathways yielding quantitative, spatially and temporally indexed information on normal and diseased tissues such as tumors. Because of its non-invasive nature, nuclear techniques also enable to repeat studies in a single subject, facilitating longitudinal assay designs of rodent models of human cancer, cardiovascular, neurological and other diseases over the entire process, from the inception to progression of the disease, and monitoring the effectiveness of treatment or other interventions (with each animal serving as its own control and thereby reducing biological variability). This also serves to reduce the number of experimental animals required for a particular study. In contrast to cell or tissue culture-based experiments, studies in intact animals incorporate all the interacting physiological factors present in vivo.
\end{abstract}

DOI: $10.12693 /$ APhysPolA.127.1483

PACS: 87.57.uk, 87.85.Pq, 87.57.un

\section{Introduction}

Imaging has long been indispensable in clinical practice. Gradually, in vivo imaging of small laboratory animals has emerged as a critical component of preclinical biomedical research as well. Imaging provides a bridge from animal research to human research and the clinic, enabling similar and sometimes identical experiments to be carried out across species. The clinically translatable, noninvasive and quantitative nature of small-animal imaging makes it an invaluable component of modern biomedical research.

Small animal imaging is the preclinical component of the new emerging field of molecular imaging aiming at non-invasive visualization, characterization and measurement of normal, as well as pathological/abnormal biological processes at the molecular or cellular level in humans. Non-invasive in vivo validation of the candidate drugs and probes is critical prior to perform human trials.

The non-invasive nature enables one to repeat studies in a single subject, facilitating longitudinal assay designs of animal models of human diseases over the entire process, from the inception to progression, and monitoring the effectiveness of treatment or other interventions (with each animal serving as its own control and thereby reducing biological variability). This also serves to reduce the number of experimental animals required for a particular study. In contrast to in vitro and ex vivo, studies in intact animals incorporate all the interacting physiological factors present in vivo $[1,2]$.

The mouse is the most used, followed by rat. The mouse makes an excellent model for human disease

\footnotetext{
* corresponding author; e-mail: marta.oteo@ciemat.es
}

because the organization of their DNA and the way their genes are expressed are very similar to humans. They are very prolific, inexpensive to house, their reproductive and nervous systems are like those of humans, and they suffer from many of the same diseases. Due to big advances in mouse genomics a wide range of animal models of human disease have been developed. In some disciplines, particularly in neuroscience, the rat also remains an important experimental animal because of its larger brain size which makes easier surgical manipulation and visualization of its different structures $[1,2]$.

\subsection{Challenges for small animal imaging}

The most obvious challenge for small animal imaging is to visualize anatomical structures and monitor physiological activities on such small scale, so high resolution imaging modalities are required [3-6]. Resolution, which is defined as the distance where two infinitely small point sources can still be distinguished from each other, affects not only the level of detail that can be seen in an image, but also can strongly affect quantification of radionuclide concentrations. But usually improved resolution is achieved at the expense of sensitivity which is translated in poorer signal-to-noise ratios. That's why "reverse translation" of clinical imaging modalities to small animal research requires substantial improvements in the different imaging modalities which involve re-engineering of many aspects of their hardware, firmware and software.

\subsection{Imaging modalities}

Some of the different small animal imaging modalities available can be used to investigate a wide range of biological processes and are therefore "functional" imaging techniques: optical imaging and nuclear techniques (positron emission tomography, PET, and single photon emission computed tomography, SPECT) while others 
such as magnetic resonance (MR), computed tomography (CT), and ultrasound (US) provide exquisite high resolution anatomic images and are often characterized as "structural" imaging modalities. Modern MR imaging methods (MRSI = spectroscopy + imaging) can also provide functional information, although sensitivity levels are in milli- to micromolar range whereas nuclear methods and optical imaging provide sensitivity levels beyond nanomolar range. Radiations in the optical and near infrared regions (NIR) of the electromagnetic spectrum, which are highly attenuated by tissue and therefore generally not useful for in vivo imaging of subjects as large as humans, but have been successfully adapted to imaging small animals. Planar optical and NIR images are only semiquantitative because measured signal is dependent on tissue depth. Although they have the advantage of relative low cost without involving the use of radioactivity, they have lower spatial resolution than nuclear techniques $[2,6]$.

Each modality has particular characteristics, advantages and limitations and they are generally considered complementary rather than competitive performance parameters and logistical features of small-animal imaging modalities and a comparative spatial resolution between clinical and preclinical imaging modalities and associated design refinements can be found in [2]. The choice of a certain imaging modality depends primarily on the specific question to be addressed. There is a consensus among experts in the field that the most sensitive techniques are the nuclear ones (PET and SPECT).

\section{PET scanners for animal research}

The goal in the designing of small animal PET scanners is to improve resolution while maintaining high sensitivity. This can be achieved by improvements in the detector instrumentation and overall system design by reducing the detector size and ring diameter, new scintillators and the use of iterative reconstruction methods. Resolution and sensitivity are also affected by radiotracer characteristics as positron range and radiotracer specific activity. Positron range in tissue (being a function of the kinetic energy with which it is emitted from the nucleus) limits the spatial resolution and significant increase in radiotracer specific activity would allow more activity to be injected into an animal, and therefore improve counting statistics and signal-to-noise ratio images. Typical preclinical PET scanners exhibit a spatial resolution in the order of $1.5-2.5 \mathrm{~mm}$ measured at full-width at half maximum (FWHM) of the point spread function in the central field of view. Most of the commercial preclinical scanners are based on small individual scintillators coupled to photomultipliers [4, 5, 7-9].

Preclinical imaging studies using PET offer the opportunity for direct translational research in molecular imaging and drug discovery from bench-to-bedside. PET can be used to characterize the pharmacodynamics and pharmacokinetics of the new molecular imaging probes and labeled drugs. New, in vitro tested, molecular imaging probes and drugs are screened and evaluated in small number of rodent models by PET, along with traditional pharmacological, biochemical and behavioral experiments. This is followed by initial studies in patients and if the results in mice can be transferred to the human situation, larger groups of animals can be examined. Finally, investigations in an expanded population of patients can be performed. In summary, preclinical imaging allows us to achieve quicker translation to clinical practice providing a scientific bridge between basic and clinical science, better scientific foundation, more rapid elimination of ineffective compounds, reduced number of experimental animals and a lower cost [4].

\subsection{Imaging probes}

Up to now, a wide array of compounds labeled with positron emitting radionuclides is available for small animal PET studies. These compounds include those measuring glucose utilization $\left({ }^{18} \mathrm{~F}-\mathrm{FDG}=\right.$ fluoro-2-deoxy2-D-glucose) receptor binding, substrate metabolism (e.g. $11 \mathrm{C}$-acetate), mineralization (e.g. ${ }^{18} \mathrm{~F}$-fluoride), blood flow (e.g. ${ }^{13} \mathrm{~N}$-ammonia), hypoxia (e.g. ${ }^{18} \mathrm{~F}-\mathrm{FMISO}$ $=$ fluoromisonidazole), protein synthesis (e.g. ${ }^{11} \mathrm{C}-\mathrm{MET}$ $=$ methionine), apoptosis, angiogenesis, enzyme activity (e.g. ${ }^{18} \mathrm{~F}-\mathrm{FLT}=$ fluorothymidine), apoptosis, angiogenesis, gene expression etc. $[1,2,7,10]$.

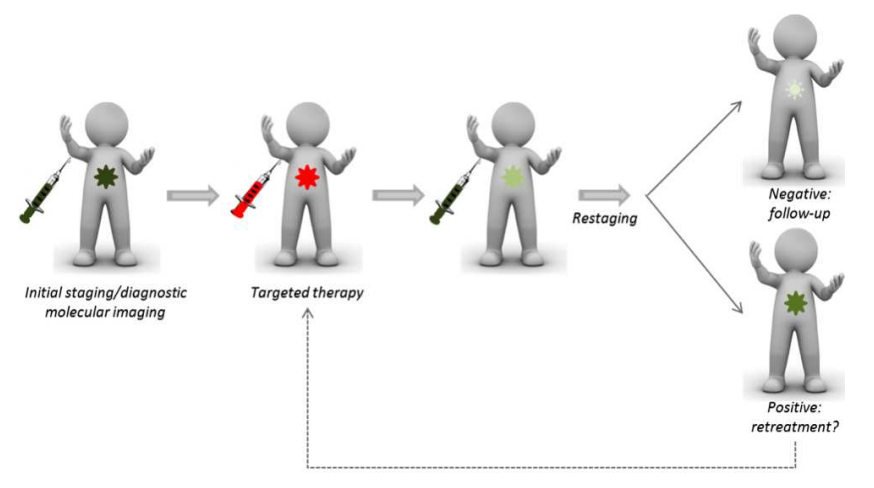

Fig. 1. Theranostics combines initial staging with an imaging version of specific probe such as radiolabeled peptides or monoclonal antibodies (green syringe), followed by therapy with the therapeutic version of the probe (red syringe). Restaging exams are performed with the imaging probe. Patients could be again treated with the therapeutic probe (positive result) or not be treated (negative result).

Nowadays, the main goal in the field of radiotracer development is the identification of specific probes for targeting cellular structures (e.g. tumor specific structures not expressed in normal cells). Radiolabeled peptides and monoclonal antibodies are ligands that can be used for diagnosis as well as for treatment, a concept known as "theranostics" (Fig. 1). If the diagnostic scan is positive they can be labeled with therapeutic radionuclides. 
The major advantages of peptides are that they have fast clearance, they are not immunogenic, show fast diffusion and target localization and they can be modified concerning metabolic stability and pharmacokinetics. Compared with small molecular weight compounds they are more tolerant with respect to modification necessary for labeling (introduction of chelating systems for radio metals). On the other hand, monoclonal antibodies (mAb) are very specific, showing low blood clearance, with optimal target/background ratios 2-4 days post injection but they have the disadvantage of being immunogenic.

Regarding to the labeling strategies, physical halflife $\left(T_{1 / 2}\right)$ of the positron emitter should be compatible with the time needed for a probe to achieve optimal target/non-target ratio. The labeling can be divided in direct for halogens, like ${ }^{18} \mathrm{~F}$ and ${ }^{124} \mathrm{I}$, or indirect labeling using chelating agents for radio metals like ${ }^{68} \mathrm{Ga}$, ${ }^{64} \mathrm{Cu}$ or ${ }^{89} \mathrm{Zr}$. The chelating agent is attached to the peptide/protein and this complex is incubated with the radiometal.

${ }^{68} \mathrm{Ga}$ labeled peptides are a new class of radiopharmaceuticals showing fast target localization and blood clearance. The short half-life of ${ }^{68} \mathrm{Ga}$ matches the pharmacokinetics of many peptides and small molecules. Longlived positron emitters like ${ }^{89} \mathrm{Zr}$ and ${ }^{124} \mathrm{I}$ are particularly suitable for $\mathrm{mAb}$ labeling, allowing imaging at late time points for obtaining maximum information. Positron emitter with a short half-life can only be used with $\mathrm{mAb}$ fragments or in pretargeting approaches. Another important consideration in the choice of positron emitter is whether the $\mathrm{mAb}$ becomes internalized after the binding to the target antigen. When ${ }^{89} \mathrm{Zr}$ labeled $\mathrm{mAb}$ are processed, the positron emitters are trapped in the cell, while in case of the ${ }^{124} \mathrm{I}$ labeled ones internalization results in a rapid clearance of the radionuclide from the target cells. This phenomenon, called residualization should be taken in account.

\subsection{1. ${ }^{68}$ Ga labeled compounds}

Lately, there has been a significant increase in the development of ${ }^{68} \mathrm{Ga}$ radiolabeled compounds with a significant rise in the number of ${ }^{68} \mathrm{Ga}$ publications in the latest years. ${ }^{68} \mathrm{Ga}$ PET agents have significant clinical promise because the radionuclide can be produced by a ${ }^{68} \mathrm{Ge} /{ }^{68} \mathrm{Ga}$ generator on site which is a very good alternative to cyclotron-based PET isotopes. The short half-life of ${ }^{68} \mathrm{Ga}$ maintains patient dose to an acceptable level. Furthermore, due to superior resolution, ${ }^{68} \mathrm{Ga}$ PET agents could replace current SPECT agents in many applications. Some ${ }^{68} \mathrm{Ga}$ radiolabeled peptides are already clinical routine for diagnosis, e.g. ${ }^{68} \mathrm{Ga}-$ somatostatin analogs with high affinity for somatostatin receptors (SSTRs), which are over expressed in neuroendocrine tumors (NETs). There are also several new Ga68 derived compounds which are at the moment under development [11-13].

Our group has been working for several years with ${ }^{68} \mathrm{Ga}$ radiolabeled compounds. Thus, in collaboration with the Melanoma Group of the National
Cancer Research Center in Spain, we labeled with ${ }^{68} \mathrm{Ga}$ a DOTA- $\alpha$-melanocyte-stimulating hormone ana$\log$ (DOTA-Napamide) which has been shown to specifically bind to melanocortin receptors that are overexpressed on human and mouse melanoma cells and assayed melanocortin receptor-binding potency of the radiopeptide in a mouse model implanted subcutaneously with a melanoma cell line (Fig. 2).
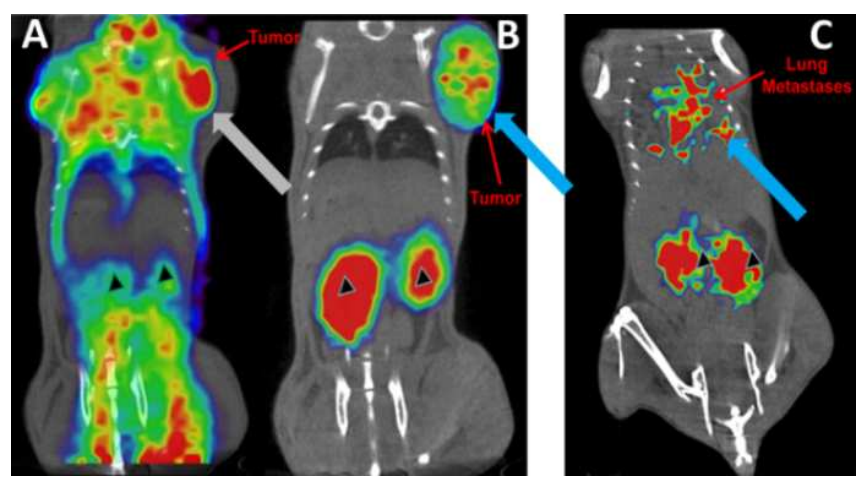

Fig. 2. ${ }^{18}$ F-FDG uptake (A) compared to ${ }^{68} \mathrm{Ga}-$ DOTA-napamide uptake (B) in subcutaneously implanted melanoma. Detection of melanoma lung metastasis is shown in $(\mathrm{C})$.

As it is shown in Fig. $2 \mathrm{~B},{ }^{68} \mathrm{Ga}$-DOTA-napamide image, there is almost no signal in other organs than the tumor; when the melanoma cell line was intravenously injected, lung metastasis could be detected (Fig. $2 \mathrm{C}$ ). ${ }^{68} \mathrm{Ga}-$ based somatostatin analogs such as ${ }^{68} \mathrm{Ga}-\mathrm{DOTA}-\mathrm{D}-\mathrm{Phe1-}$ Tyr3-octreotide ( ${ }^{68} \mathrm{Ga}$-DOTATOC), ${ }^{68} \mathrm{Ga}$-DOTA-D-Phe1Nal3-octreotide $\left({ }^{68} \mathrm{Ga}-D O T A N O C\right)$ and ${ }^{68} \mathrm{Ga}-D O T A-D-$ Phe1-Tyr3-Thr8-octreotate $\left({ }^{68} \mathrm{Ga}\right.$-DOTATATE) are currently the most common. We are using these analogs to detect tumors expressing SSTRs (such as meningioma and pheochromocytoma) in subcutaneously implanted mouse models with different cell lines since ${ }^{18}$ F-FDG has a limitation in the detection of these tumors with low grade malignancies and slow growth rates.

\subsubsection{Immuno-PET}

Immuno-PET combines the high resolution and sensitivity of a PET camera with the unique ability of a $\mathrm{mAb}$ to selectively bind specific antigens. Although immunoSPECT images were very informative, their limited resolution and the need for more accurate quantification was a reason to explore the potential of PET for $\mathrm{mAb}$ imaging. Clinically, immuno-PET makes it possible to monitor individual patients before the administration of expensive medicine, providing an opportunity to individualize mAb therapy.

The radioisotopes more suitable for immuno-PET are ${ }^{124} \mathrm{I}\left(T_{1 / 2}=103 \mathrm{~h}\right)$, which is the radionuclide of choice in combination with non internalizing $\mathrm{mAb}$, and radiometals such as ${ }^{89} \mathrm{Zr}\left(T_{1 / 2}=78.4 \mathrm{~h}\right)$ and ${ }^{64} \mathrm{Cu}\left(T_{1 / 2}=12.7 \mathrm{~h}\right)$. Labeling with radiometals requires premodification of the $\mathrm{mAb}$ with a bifunctional chelating agent and as this 
indirect labeling is frequently more stable than direct labeling with a halogen, radiometals are often more suitable for imaging of internalizing targets. The physical half-life of these positron emitters is compatible with the time need for $\mathrm{mAb}$ to achieve optimal tumor-to-nontumor ratios; although ${ }^{64} \mathrm{Cu}$ cannot be used for immunoPET studies requiring long-term monitoring, it has been shown that high-contrast images using this residualizing radionuclide can be obtained as early as 1 day postinjection. As the use of $\mathrm{mAb}$ as probes for imaging purposes requires long waiting times, up to several days after administration, in order to have good signal-to-background image, a lot of antibody fragments have emerged as imaging agents.
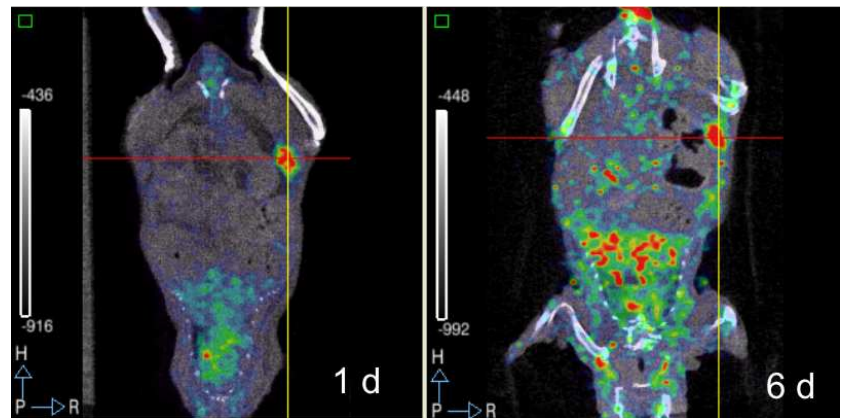

Fig. 3. Immuno-PET performed at 1 and 6 days after the injection of ${ }^{89} \mathrm{Zr}-\mathrm{DFO}-\mathrm{mAb}$ in a subcutaneously implanted mouse with a glioblastoma cell line.

These probes, due to their smaller size, clear faster from blood compared to full size antibodies and can be imaged soon after the administration which allows labeling with short-lived radioisotopes as ${ }^{68} \mathrm{Ga}$ [14-17]. Our group at CIEMAT is working in preclinical immuno-PET using $\mathrm{mAb}$ labeled with ${ }^{89} \mathrm{Zr}$. A PET scan performed at different times after the administration ( 1 and 6 days) of ${ }^{89} \mathrm{Zr}$ labeled $\mathrm{mAb}$ to a subcutaneously implanted mouse model of glioblastoma is shown in Fig. 3, where an excellent tumor tracer uptake was observed.

\section{Conclusion}

PET molecular imaging allows the non-invasive assessment of biological and biochemical processes in living subjects using different imaging probes to measure the expression of indicative markers at different stages of diseases, contributing to improve our understanding of disease and drug activity during preclinical and clinical drug development.

\section{References}

[1] S.R. Cherry, S.S. Gambhir, ILAR J. 42, 219 (2001).

[2] F. Kiessling, B.J. Pichler, Small Animal Imaging, 1st ed., Springer-Verlag, Berlin 2011.

[3] A.F. Chatziioannou, Eur. J. Nucl. Med. 29, 98 (2002).

[4] R. Myers, Nucl. Med. Biol. 28, 585 (2001).

[5] C. Kuntner, D. Stout, Front Phys. 2, 12 (2014).

[6] A.S.K. Dzik-Jurasz, Brit. J. Radiobiol. 76, S98 (2003).

[7] B. Riemann, K.P. Schäfers, O. Schober, M. Schäfers, Q. J. Nucl. Med. Mol. Imaging 52, 215 (2008).

[8] S.P. Hume, T. Jones, Nucl. Med. Biol. 25, 729 (1998).

[9] J.L. Vanderheyden, Q. J. Nucl. Med. Mol. Imaging 53, 374 (2009).

[10] M.E. Phelps, Proc. Natl. Acad. Sci. USA 97, 9226 (2000).

[11] D.L. Smith, W.A. Breeman, J. Sims-Mourtada, Appl. Radiat. Isot. 76, 14 (2013).

[12] A. Al-Nahhas, Z. Win, T. Szyszko, A. Singh, S. Khan, D. Rubello, Eur. J. Nucl. Med. Mol. Imaging 34, 1897 (2007).

[13] M. Pagou, I. Zerizer, A. Al-Nahhas, Hell. J. Nucl. Med. 12, 102 (2009).

[14] G.A. van Dongen, G.W. Visser, M.N. Lub-de Hooge, E.G. de Vries, L.R. Perk, Oncologist 12, 1379 (2007).

[15] M. Salsano, G. Treglia, Res. Rep. Nucl. Med. 3, 9 (2013).

[16] J. De Vos, N. Devoogdt, T. Lahoutte, S. Muyldermans, Expert Opin. Biol. Ther. 13, 1149 (2013).

[17] S.M. Knowles, A.M. Wu, J. Clin. Oncol. 30, 3884 (2012). 